\title{
BMJ Open Evaluating spillover of HIV knowledge from study participants to their network members in a stepped-wedge behavioural intervention in Tanzania
}

\author{
Jeffrey Rewley (D) , ${ }^{1,2}$ Mary C Smith Fawzi, ${ }^{3}$ Keith McAdam, ${ }^{4}$ Sylvia Kaaya (D) , ${ }^{5}$ \\ Yuanyuan Liu, ${ }^{6}$ Jim Todd, ${ }^{7}$ Irene Andrew, ${ }^{8}$ Jukka Pekka Onnela ${ }^{9}$
}

To cite: Rewley J, Fawzi MCS, McAdam K, et al. Evaluating spillover of HIV knowledge from study participants to their network members in a steppedwedge behavioural intervention in Tanzania. BMJ Open 2020;10:e033759. doi:10.1136/ bmjopen-2019-033759

- Prepublication history for this paper is available online. To view these files, please visit the journal online (http://dx.doi. org/10.1136/bmjopen-2019033759).

Received 21 August 2019 Revised 06 August 2020 Accepted 10 August 2020

\section{Check for updates}

(C) Author(s) (or their employer(s)) 2020. Re-use permitted under CC BY-NC. No commercial re-use. See rights and permissions. Published by BMJ.

For numbered affiliations see end of article.

\section{Correspondence to} Dr Jeffrey Rewley; jeffrey.rewley@pennmedicine. upenn.edu

\section{ABSTRACT}

Objectives We aim to describe the social network members of participants of a behavioural intervention, and examine how the effects of the intervention may spillover among network members.

Design Secondary analysis of a step-wedge randomised controlled trial.

Setting Change agents (CAs) were recruited from waiting rooms of HIV treatment facilities in Dar es Salaam, Tanzania, and their network members (NMs) were recruited directly by CAs.

Participants We enrolled 662 CAs in an HIV behavioural intervention. They, along with 710 of their NMs, completed baseline and follow-up interviews from 2011 to 2013.

Primary and secondary outcomes The primary outcome of this study was change in NMs' HIV knowledge, and the secondary outcome was whether the NM was lost to follow-up.

Results At baseline, many characteristics were different between NMs and CAs. We found a number of NM characteristics significantly associated with follow-up of $\mathrm{NMs}$, particularly female gender $(\mathrm{OR}=1.64,95 \% \mathrm{Cl}: 1.02$ to 2.63) and HIV knowledge (OR=20.0, 95\% Cl: 3.70 to 125); only one CA variable was significantly associated with NM follow-up: having a private source of water $(\mathrm{OR}=2.17$, $95 \%$ Cl: 1.33 to 3.57$)$. The $14.2 \%$ increase in NMs' HIV knowledge was largely due to CAs feeling empowered to pass on prior knowledge, rather than transmitting new knowledge to their NMs.

Conclusions Characteristics of social network members of persons living with HIV persons living with HIV may play a role in study retention. Additionally, the HIV knowledge of these NMs increased largely as a function of CA participation in the intervention, suggesting that intervening among highly-connected individuals may maximise benefits to the potential population for whom spillover can occur.

Trial registration number Clinical Trial: NCT01693458; Post-results

\section{INTRODUCTION}

Despite recent decreases in mortality, HIV/ AIDS is currently the ninth leading cause of years of life lost globally. ${ }^{1}$ Research into eliminating HIV has focussed on two fronts:
Strengths and limitations of this study

- The recruitment method, focussed on social network members of persons living with HIV, allowed us to access an at-risk population typically not easilyaccessible via other means, such as direct approach by the study team rather than someone known to the participant.

- The study design of the trial in which this study is nested was an ideal situation in which to employ a mediation analysis that informs our understanding of how this and similar intervention effects may spillover in a population.

- The greater-than-ideal dropout rate of NMs was both a strength in that it allowed us to examine factors associated with dropout, but also a limitation, in that the potential of differential dropout by unmeasured factors may have biassed some of our results.

biological and behavioural, which combined have decreased the years of life lost due to HIV by an estimated $51 \% .^{1}$ Behavioural interventions seek to curb transmission of HIV through reduction of risk behaviour including safer sexual practices and reducing injection drug use. ${ }^{2}{ }^{3}$ Behavioural change also impacts the effectiveness of the biological methods, given that lower adherence is known to often compromise their effectiveness. ${ }^{4}$ However, neither biological nor behavioural methods are fully effective without the knowledge of these treatments and preventative behaviours. It is therefore important to increase HIV-related knowledge as a necessary, but not sufficient, step towards achieving the 90-90-90 treatment goals (90\% diagnosis, $90 \%$ antiretroviral therapy and $90 \%$ viral suppression among the treated) and ultimately eliminate $\mathrm{HIV}^{5}$

The importance of adequate HIV knowledge has been recognised for a period of time, as it is often a prerequisite for behavioural 
change. ${ }^{6}$ It should be noted that increased knowledge does not necessarily translate into behaviour change, as other factors such as motivation and skills also play a role. ${ }^{6}$ Despite this, researchers conducted trials among persons living with HIV (PLH) which led to increased HIV knowledge. ${ }^{78}$ These interventions were group discussions and teaching led by nurses, and motivational interviewing, respectively. Researchers also found that the intervention reduced HIV-risk behaviours concomitantly with an increase in HIV knowledge. ${ }^{8}$

Knowledge gained by participants in these trials can also be freely shared with members of their social network, in what is known as a spillover effect. ${ }^{9}$ Specifically, a spillover effect (also known as an indirect or disseminated effect) is one person's exposure affecting another's outcome. ${ }^{10}$ In the context of a social network spillover intervention, it corresponds to an individual who was unexposed to an intervention changing their behaviour because they were socially-connected to an individual who did receive the intervention. This is distinct from what is sometimes called behavioural spillover, where changes in a person's behaviour affect other behaviours of that same person. ${ }^{11}$ For injection drug users, HIV prevention educational interventions were demonstrated to have spillover effects of HIV prevention education, and subsequent reduced rates of risky behaviours. ${ }^{12}$ Studies have also used proxy variables for social network ties such as inviting social network members to watch educational programming ${ }^{13}$ or time spent shopping at the market ${ }^{14}$ to evaluate spillover effects for HIV knowledge, generally finding evidence for spillover. However, spillover in HIV knowledge between known social network ties generally remains understudied, particularly in sub-Saharan Africa. ${ }^{15}$ We therefore aim to determine whether social network members those receiving an HIV behavioural/knowledge intervention also increase their HIV knowledge.

New knowledge can come from a variety of sources, one of the most important of which is a person's social network. ${ }^{16}$ Social networks are of particular import because new knowledge can lead to cascades of behaviour change, where people subsequently educate those in their social network, in what is known as social influence. ${ }^{18-20}$ This has been examined in participant-driven interventions, where initially-recruited participants educate members of their social network one-on-one. ${ }^{21}$ Characteristics such as knowing the HIV status of network members has been shown to be the most important predictor of engaging in prevention advocacy. ${ }^{22}$ Work on diffusion through social networks, how a belief or behaviour can be 'contagious' within a network, has shown that spreading intervention effects beyond the initial study population can improve the cost-effectiveness of these interventions. ${ }^{23}$ These findings imply that certain aspects of knowledge or behaviour may spread more or less efficiently through networks comprising individuals with specific characteristics, which may need to be accounted for in network interventions. For instance, networks comprising many at-risk individuals who are HIV-negative may not be as receptive to change in behaviours as networks comprising a mix of those with and without HIV. Additionally, the networks of PLH are often difficult networks to ascertain, ${ }^{24}$ due to the continued stigma of HIV and AIDS in many settings. ${ }^{25}$ Because of this, if a PLH or person at-risk of acquiring HIV does not want to participate in an investigator-initiated intervention, there is little recourse other than information transmitted via social networks, or targeted sampling techniques which are not always effective (eg, Respondent Driven Sampling). ${ }^{26}$ This is particularly important in low-income and middle-income countries, as a recent systematic review found only 54 studies researching spillover effects in these settings (out of approximately 750). ${ }^{13}$ Therefore, understanding exactly how information spreads from participants in an intervention to members of their social network, who may be largely inaccessible via other means, is important for reaching the greatest number of people about HIV prevention. Understanding what makes these persons different from those who receive the intervention themselves is important, as it may point to ways in which to increase enrolment of these populations.

Based on the above gaps in the literature, we conduct a study on network members of PLH enrolled in a behaviour change intervention. ${ }^{27}$ The trial recruited PLH to serve as change agents (CAs) and to reach out to their social network members (NMs) about knowledge of HIV and safer sexual practices. ${ }^{28}$ Our goals in this study were threefold: (1) to understand how the NMs differed from their CAs, (2) understand correlates of dropout for the NMs and (3) to understand by what method HIV knowledge transferred to the NMs from the CAs. Understanding how the information and behaviours are shared within social networks will allow HIV researchers and others to take advantage of this knowledge and improve on prevention interventions in the future.

\section{METHODS}

\section{Study population}

We analyse social network data from the Agents of Change trial,${ }^{29}$ which was a stepped-wedge randomised controlled trial $^{30}$ that enrolled PLH to become CAs by informing members of their social NMs about knowledge of HIV and safer sexual practices. Here, we define CAs based on the potential for PLH to become so-by self-selecting into the study, PLH identify themselves as potential CAs. Although we refer to them as 'CAs' throughout, participants in the trial enrolled with varying levels of ability to act as a CA. Through receiving the NAMWEZA intervention, we hypothesise that CAs will be able to truly selfactualise and subsequently act as CAs in their community.

CAs were recruited from the waiting rooms of HIV care and treatment centres in Dar es Salaam, Tanzania, and we received written consent from each CA. Participants completed a baseline questionnaire and were randomised to one of three waves in which to receive the intervention. At baseline, participants were also asked to recruit up to 
three members of their social networks who they felt were at particularly high risk of contracting or spreading HIV. We obtained written consent from these nominated NMs. NMs could be either HIV positive or negative, and they were given a baseline survey. The NM was only aware that the CA was a participant in the intervention if the CA shared this information with them, which many did not due to HIV-related stigma. ${ }^{31}$ Each CA therefore formed a CA-NM dyad with each NM they recruited, and if they recruited more than one NM, formed a set of CA-NM dyads with a common CA.

As fits a stepped-wedge randomisedcontrolled trial, all CAs eventually received the intervention, but were randomised to when they received it. These waves each lasted 12 weeks, at which point the next wave began and another group of CAs received the intervention. Within each wave, the intervention comprised 10 weekly structured sessions aimed at empowering PLH to become HIV prevention change agents in their communities. The sessions aimed to increase psychosocial and communication skills through an Appreciative Inquiry approach. ${ }^{32}$ Within 1 month of each wave of the intervention, CAs were given follow-up surveys. Across all waves, the interventions lasted from November 2010 to January 2014, and the final interviews were conducted in March 2014. For more information on the study design, we direct interested readers to Smith Fawzi et al, 2019. ${ }^{28}$

The NMs did not receive any intervention at any point during the study. Rather, their intervention status flowed from the intervention status of their CAs. Due to operational difficulties, each NM was surveyed two times during the study: baseline and after the first wave, rather than baseline and one after each wave. In this way, all demographic and contextual variables were measured at baseline. We use this interview of NMs as the division between 'exposed' and 'unexposed'. At the time of an NM's follow-up interview, their respective CA may or may not have undergone the intervention. In other words, the CAs randomised to receive NAMWEZA during the first wave would have potentially indirectly exposed their NMs to the intervention when the NM completed their follow-up questionnaire after Wave 1. Therefore, the NMs were divided into 'exposed' ( $\mathrm{n}=381)$ and 'unexposed' ( $n=329$ ) groups based on whether their respective CA was randomised into receiving NAMWEZA during the first wave or not. The CAs always completed their Wave 1 follow-up interview before their NMs were invited to complete their Wave 1 follow-up interview.

Inclusion criteria for the follow-up analyses were for CAs who completed at least one follow-up interview and had at least one NM who completed at least one follow-up questionnaire. In this way, outcomes could be computed for the CA and NM of each dyad. NMs approached by a CA, but who did not participate in the study were not recorded since it was not feasible to obtain this information from study participants themselves. As we lost some CAs and NMs to follow-up, we completed our analyses without their data, assuming it to be missing completely at random. During this study, there was little loss-tofollow-up among the CAs $(<10 \%)$, but much higher loss among the NMs (36.8\%). ${ }^{33}$ Given an NM or CA was not lost to follow-up, complete information was available on all additional variables, including exposure, outcome and covariates. In sum, our sample comprises 662 CAs and $710 \mathrm{NMs}$, meaning each CA recruited $1.07 \mathrm{NMs}$ on average out of a possible 3 , and 44 CAs nominated at least 2 NMs.

\section{HIV knowledge}

To assess HIV knowledge of CAs and NMs, we used the brief HIV knowledge questionnaire. ${ }^{34}$ This scale comprises 18 questions, which focus on an individual's knowledge of how HIV can spread and other characteristics of the virus and AIDS. This knowledge is crucial for an individual's subsequent safe-sex practices to reduce the risk of transmission. The original population comprised three different groups: two groups of low-income women, and one of women and men receiving psychiatric treatment. In these populations, questions on the measure had a Chronbach's alpha of 0.78. This instrument has been used previously in sub-Saharan Africa and has demonstrated reasonable reliability of 0.75 Cronbach's alpha in South Africa among a convenience sample of 429 members of the African Methodist Episcopal church. ${ }^{35}$ It has also been translated to Swahili, with only minor differential item functioning. ${ }^{36}$ This indicates that the measure performs adequately in other, similar populations. In the present study the Cronbach's alpha is 0.71 . Because the average baseline score of HIV knowledge was 0.80 , with a preponderance of scores of 1.00 , the main indicator of knowledge was not normally distributed, and therefore a continuous predictor was not ideal. ${ }^{37}{ }^{38}$ We therefore summarise this measure as 'Complete HIV Knowledge', a dichotomous variable for whether the participant correctly answered all questions.

\section{Demographic and contextual variables}

In addition to HIV knowledge, we are interested in a set of demographic and contextual variables that may help explain some of the trends observed. In terms of demographic variables, we include age, sex, employment status, marital status and self-identified HIV status. We also include education, which we dichotomise at 7 years or more-a level commensurate with elementary education. We selected this cut-off because it coincides with the millennium development goal (MDG) of increasing primary education completion. ${ }^{39}$

Contextually, we included two additional variables: having a private source of water, and the number of persons sleeping in the participant's home. The first was also based on a MDG, and indicates participants with access to safe drinking water. ${ }^{39}$ This is a proxy for the economic security of the participant. The number of persons sleeping in the participant's home is also a non-monetary indicator of their material and social 
resources. ${ }^{40}$ These variables combined give a more thorough picture of the participant's economic status than employment alone.

\section{Statistical analysis}

The first analysis was to assess homophily, defined as the extent to which CAs and NMs were similar to one another in terms of a number of sociodemographic characteristics and HIV-related risk factors. Because CAs and NMs self-selected into their respective group, and only CAs were randomised (with their NMs being randomised along with them), differences between the groups were to be expected. However, we only examined homophily of baseline characteristics rather than of outcome, because comparison of outcomes between CAs and NMs would remove the benefits of randomisation. Because CAs and NMs self-selected into the study and were not randomised to $\mathrm{CA} / \mathrm{NM}$ status, we do not a priori expect them to be completely similar. In addition, multiple NMs could share a CA and would therefore not be independent due to the shared variation and latent characteristics of having the same CA. We therefore assessed statistical significance of homophily on the set of CA-NM dyads using a permutation test, a non-parametric test which has no distributional assumptions. For continuous variables, the difference between the CA and NM was calculated, and for categorical variables, whether the CA and NM were concordant or discordant was recorded. We then randomly permuted CA-NM ties (keeping number of ties per CA constant), and then recalculated the difference or percent concordant, respectively, 1000 times. We then examine the percentile of the observed difference relative to the permuted differences. ${ }^{41}$ Analyses were run using R V.3.1.1.

To accomplish our second aim of understanding what was associated with NMs completing their follow-up interview, we fit a log-binomial regression to determine predictors of follow-up. In this regression, we use all the variables listed above, as well as whether the CA remained in the study for its full duration. In order to examine the association between these same variables and the time of follow-up, we also employed a Cox regression. In the Cox regression, start of follow-up was defined as the time at which the NM completed their baseline interview. The outcome here was whether the NM completed a follow-up interview. NMs who were lost to follow-up were censored at the time of their latest interview. ${ }^{42} 43$

Finally, since the trial showed beneficial effects on the HIV knowledge of the NMs following the intervention, ${ }^{29}$ we aim to elucidate exactly what caused the HIV knowledge of NMs to increase-either CAs gaining knowledge through the intervention and sharing it, or the CAs being empowered by the intervention to share existing knowledge. As the wedge in which the CA received the NAMWEZA intervention was randomised, we treat each $\mathrm{NM}$ as being randomised to exposure to NAMWEZA at the same time as their CA. This randomisation scheme allows us to explore the spillover effect of CAs' HIV knowledge
A: Intervention status of the CA YCA: HIV knowledge of the $\mathrm{CA}$ at the interview immediately prior to the NM's follow-up interview $Y_{N M}$ : HIV knowledge of the NM at follow-up

Figure 1 Schematic of natural direct effect (NDE) and natural indirect effect (NIE). The NDE indicates the increase in NMs' HIV knowledge happens without a concomitant increase in their CA's HIV knowledge. The NIE, on the other hand, indicates that the increase in NMs' HIV knowledge is mediated by their CA's HIV knowledge increasing. Solid lines indicate paths of causality between variables. Dashed lines represent the line or lines composing the effect of interest. $\mathrm{CA}$,change agent; NM, network member.

onto their respective NMs via a mediation analysis. These pathways represent different types of spillover effects: the exposure or outcome of one person affecting the outcome of another person.

As shown by VanderWeele et al (2015), social network spillover effects in the case of dyadic relationships can be broken down into concepts from mediation analysis: direct and indirect effects (figure 1). ${ }^{44}$ This method has since been used for novel evaluations of spillover effects. ${ }^{45-47}$ Although previous studies showed that this same type of analysis cannot be done on full network data, the data in this study consisted of only dyads, the CAs and their NMs, so in this case the method is appropriate. ${ }^{48} 49$

The method parses social influence into direct and indirect effects. The natural indirect effect (NIE) is the effect by which the intervention changes the CA's HIV knowledge, which then changes the NM's HIV knowledge. The NIE is similar to the effect observed in many participant-driven interventions: an initial participant receives the intervention, increasing their knowledge and they subsequently pass their increased knowledge to members of their social network. ${ }^{21}$ The natural direct effect (NDE) is the effect of receiving the intervention has on an NMs outcome, irrespective of the CA's outcome (in this case HIV knowledge). For instance, this could occur if CAs begin with good knowledge of HIV, and the intervention empowers them to convey knowledge they already had to their NMs. Although the intervention does not increase their HIV knowledge, it is still useful to the CAs, as it empowers them to act as CAs in their community. In order to estimate these effects, the published SAS macro developed by Vanderweele and colleagues was used, and analyses were run using SAS V.9.2 (SAS Institute, Cary, North Carolina). ${ }^{50} 51$ In the models estimating the effect of the exposure on the mediator and estimating the effect of the mediator on the outcome, we adjusted for all the variables included in our logistic regression above. Although the randomisation of the exposure minimised some potential bias, the loss-to-follow-up among the CAs indicates that selection bias could remain a concern, so we control for the variables which may also impact loss-to-follow-up. 
Importantly, this analysis requires a number of assumptions and applies to dyads only when these assumptions are met. One assumption of this analysis is that the dyads are independent, which is violated here; if a CA recruited more than one NM, the multiple CA-NM dyads involving the same CA would not be independent. To address this, we performed the analysis after randomly removing NMs until each CA had only a single NM. This resulted in removing $48 \mathrm{NMs}$, just $6.7 \%$ of the population. We found that the point estimates were nearly identical, but that the CIs were slightly larger due to the reduced sample size. No coefficients changed from significant to nonsignificant in this analysis (data not shown).

A second, related assumption is that of partial interference, that the effects in one cluster does not affect another cluster-here, one CA-NM dyad affecting another. ${ }^{52}$ This could occur if two NMs of different CAs happen to know one-another outside of the study, one has a CA who was randomised to an earlier wedge, and shares what they know of it with the otherwise-unexposed NM. However, due to the large size of Dar es Salaam, and the number of HIV treatment clinics in which recruitment occurred, we expect few CAs or NMs to know one another outside of the study (other than NMs knowing the CA who recruited them), limiting the potential for partial interference.

A third assumption of this analysis is that the outcomes of the CA and NM are independent conditional on the CA's exposure, or conditional on the CA's exposure and any confounding variables. ${ }^{445}$ Because CA-NM pairs selfselect and are not randomised, we do not expect these outcomes to be independent conditional of the CA's exposure, and so we adjust for additional variables to meet this assumption. In our analysis, we therefore adjust for all the variables used in the log-binomial regression.

The data are not publicly available due to the sensitive nature of HIV infection status and the socially-networked nature of the data. Because the data include specific information on social ties, some of whom have not disclosed HIV sero-status to one another, the risks of individual identification and compromising HIV sero-status are greatly increased.

\section{Patient and public involvement}

Patients were not involved in the design of this study. As part of the recruitment process, patients were instructed to recruit members of their social networks who they felt were at high risk of HIV infection-in this way participants could guide the recruitment of NMs to those most at-risk. We currently have no plans to disseminate the results of this study to participants.

\section{RESULTS}

The NAMWEZA study recruited 662 CAs, of whom 453 $(68.4 \%)$ completed at least one follow-up questionnaire. These 662 CAs in turn recruited 710 NMs who took the baseline questionnaire. Of the $710 \mathrm{NMs}, 449$ (63.2\%) also completed a follow-up questionnaire (table 1). At
Table 1 Demographic characteristics at baseline, with the results of a permutation test for homophily

\begin{tabular}{|c|c|c|c|}
\hline Characteristic & $\begin{array}{l}\text { Number of } \\
\text { NMs (\%) or } \\
\text { mean (SD) } \\
(n=710)\end{array}$ & $\begin{array}{l}\text { Number of } \\
\text { CAs (\%) or } \\
\text { mean (SD) } \\
(n=662)\end{array}$ & $P$ value \\
\hline Age & $33.0(11.1)$ & $38.9(9.7)$ & $<0.001$ \\
\hline Female & $380(53.7 \%)$ & 349 (53.9\%) & 0.89 \\
\hline Employed & $490(69.3 \%)$ & 356 (55.0\%) & $<0.001$ \\
\hline $\begin{array}{l}\text { At least } 7 \text { years } \\
\text { education }\end{array}$ & 369 (52.0\%) & $584(82.3 \%)$ & $<0.001$ \\
\hline $\begin{array}{l}\text { Complete HIV } \\
\text { knowledge }\end{array}$ & $638(89.9 \%)$ & $598(90.4 \%)$ & 0.65 \\
\hline $\begin{array}{l}\text { Persons sleeping } \\
\text { in home }\end{array}$ & $5.00(5.77)$ & 3.87 (3.72) & $<0.001$ \\
\hline Married & $373(52.7 \%)$ & 338 (51.1\%) & 0.56 \\
\hline HIV positive & 87 (12.3\%) & $662(100 \%)$ & $\mathrm{N} / \mathrm{A}$ \\
\hline $\begin{array}{l}\text { Private source of } \\
\text { water }\end{array}$ & 309 (43.7\%) & $263(39.7 \%)$ & 0.19 \\
\hline
\end{tabular}

Specifically, for each CA-NM dyad, either a difference (for continuous variables) or concordance (for dichotomous variables) is calculated. For example, if a changeagent (CA) was 39 years old, and their networkmember (NM) was 25 years old, the difference would be 14 years old. If a CA was male and their NM was female, the pair would be discordant for sex. CA-NM pairs were then randomly reshuffled, the edge-wise characteristics recalculated, and the observed difference compared to the distribution of randomised differences.

baseline, CAs were on average older than their NMs. More of the NMs were employed than their CAs $(69.3 \%$ vs $55.0 \%, \mathrm{p}<0.001)$, but were less likely to have at least 7 years of education $(52.0 \%$ vs $52.3 \%, \mathrm{p}<0.001)$. Only $12.3 \%$ of NMs were HIV-positive, compared with all CAs $(p<0.001)$. Complete data was obtained at baseline for all CAs and NMs.

Risk ratios (RRs) obtained via log-binomial regression indicated that characteristics of both the CAs and the NMs significantly predicted loss to follow-up (table 2). The NM being female ( $R R=1.44,95 \%$ CI: 1.05 to 1.97$)$, having complete HIV knowledge ( $\mathrm{RR}=10,95 \%$ CI: 2.33 to 42 ), being employed ( $\mathrm{RR}=1.43$, $95 \%$ CI: 1.08 to 1.89$)$, and being married ( $R R=1.55,95 \%$ CI: 1.03 to 2.33 ) were all significant predictors of increased odds of completing a follow-up interview. Each additional person sleeping in the home of the NM per room used for sleeping reduced the odds of follow-up ( $\mathrm{RR}=0.85,95 \%$ CI: 0.74 to 0.98 ) as did the NM living with HIV ( $\mathrm{RR}=0.40,95 \%$ CI: 0.17 to 0.96$)$. CA having a private water source was significantly associated with increased odds of the NM being followed-up ( $R R=2.07,95 \%$ CI: 1.25 to 3.42 ), even after controlling for the NM having a private source of water. This was the only CA-specific variable that significantly predicted an NM's follow-up. 
Table 2 Results of multivariate log-binomial regression and Cox proportional hazard models on the dichotomous outcomes of whether the NM completed a follow-up questionnaire, and the continuous outcome of time-to-completion of follow-up questionnaire, respectively

\begin{tabular}{|c|c|c|}
\hline Characteristic $(n=459)$ & Adjusted RR (95\% Cl) & Adjusted HR (95\% CI) \\
\hline \multicolumn{3}{|l|}{ Characteristics of NMs } \\
\hline Gender (female) & $1.44^{*}(1.05$ to 1.97$)$ & 1.18 (0.94 to 1.50$)$ \\
\hline Difference in age of NM and CA (per year) & 1.01 (0.99 to 1.03$)$ & 1.01 (0.995 to 1.02$)$ \\
\hline Age of NM & 0.98 (0.95 to 1.02$)$ & 0.99 (0.97 to 1.04$)$ \\
\hline Complete HIV knowledge (vs none) & $10^{*}(2.33$ to 42$)$ & 2.20 (0.97 to 5.01$)$ \\
\hline Employed & $1.43^{\star}(1.08$ to 1.89$)$ & 1.15 (0.89 to 1.50$)$ \\
\hline Each additional person sleeping in home & $0.85^{\star}(0.74$ to 0.98$)$ & $0.92(0.83$ to 1.01$)$ \\
\hline \multicolumn{3}{|l|}{ Per room used for sleeping } \\
\hline Married & $1.55^{\star}(1.03$ to 2.33$)$ & $1.28^{*}(1.01$ to 1.64$)$ \\
\hline Living with HIV & $0.40^{\star}(0.17$ to 0.96$)$ & $0.71^{\star}$ (0.51 to 0.99$)$ \\
\hline Having a private source of water & 0.89 (0.59 to 1.34$)$ & 0.97 (0.77 to 1.22$)$ \\
\hline \multicolumn{3}{|l|}{ Characteristics of CAs } \\
\hline Gender (female) & 1.27 (0.76 to 2.08$)$ & 1.07 (0.84 to 1.37$)$ \\
\hline Complete HIV knowledge (vs none) & 0.36 (0.07 to 2.04$)$ & 0.64 (0.29 to 1.43$)$ \\
\hline Having a private source of water & $2.07^{*}(1.25$ to 3.42$)$ & 0.97 (0.77 to 1.22$)$ \\
\hline Being employed & 1.54 (0.95 to 2.50$)$ & $1.14(0.90$ to 1.43$)$ \\
\hline Being married & 1.25 (0.77 to 2.04$)$ & 1.11 (0.88 to 1.43$)$ \\
\hline CA lost to follow-up & 1.06 (0.84 to 1.33$)$ & 1.02 (0.72 to 1.43$)$ \\
\hline
\end{tabular}

*indicates significance at the $\mathrm{p}<0.05$ level.

$\mathrm{CA}$, change agent; NM, network member; RR, risk ratio.

The Cox proportional hazard model showed similar results to the logistic regression, but with fewer significant results. Only the NM being married ( $\mathrm{HR}=1.28,95 \%$ CI: 1.01 to 1.64$)$ and the NM living with HIV (HR=0.71, 95\% CI: 0.51 to 0.99$)$ significantly predicted the time-tofollow-up of the NMs. Additionally, all of the hazard ratios were closer to the null relative to the corresponding ORs.

We assess the mechanism of spillover effects on HIV knowledge, the main effect of the intervention on the NMs. The analysis showed that NMs who were exposed to intervention via their CA had an increase in HIV knowledge of $12.9 \%$ on average (95\% CI: 0.06 to 0.20 ) from a baseline percentage of $78 \% .{ }^{26}$ This was broken down into a NIE of $0.6 \%$ (95\% CI: $-0.06 \%$ to $2.0 \%$ ), which is the effect the intervention had via the CA's HIV knowledge, and a NDE of $12.3 \%$ (95\% CI: $6.1 \%$ to $19.3 \%$ ), which is the effect the CA's participation had on the NM's HIV knowledge, irrespective of the CA's HIV knowledge. In other words, of the $12.9 \%$ increase in NM's having complete HIV knowledge, $12.3 \%$ occurred without a concomitant increase in their CA's HIV knowledge. In other words, their HIV knowledge increase was not mediated by the increase in HIV knowledge of their CA. This did not change when we used only one NM per CA.

\section{DISCUSSION}

Based on results from the NAMWEZA trial in Tanzania, we have shown a number of novel findings regarding the network members of HIV intervention participants, correlates of their retention in the study and the evidence for spillover of HIV knowledge from CAs to NMs. These findings can inform the design of interventions in the future to maximally enrol and retain participants and ensure that intervention information is transmitted from the study participants to members of their social networks.

We found several significant differences between CAs and NMs they recruited. On average, CAs were older, less likely to be employed and more educated. All of this suggests that by having CAs recruit from their social network, we were able to recruit a set of social network members different in multiple ways from those found in the waiting rooms in HIV treatment clinics (unsurprising, given that many NMs were not HIV-positive) ${ }^{9}$ This may in part be due to the fact that when recruiting NMs, CAs were instructed to recruit those who may be at particular risk of contracting or spreading HIV. Therefore, CAs likely did not pick random members of their social network, but those who were at high risk. This finding corroborates the claim by Latkin $e t a \vec{p}^{3}$ that sampling via enumeration of network members by initial participants recruits a more diverse sample, as our sample of NMs was not composed of only those who were HIV-positive, but also many who were HIV-negative. Additionally, the lower likelihood 
of employment of the CAs indicates they may have had more time for participating in the intervention. ${ }^{54}$ This means that future studies may need to tailor their interventions to work with the schedules of employed persons to increase participation.

Although the study design potentially accessed a separate slice of the population than other methods vis-a'-vis the population of those at risk for contracting HIV, it may have been at least partially responsible for the large loss of follow-up of the NMs. It is important to note that more NM characteristics than CA characteristics were significantly predictive of follow-up in both the logistic and Cox proportional hazards models. The one CA characteristic which did predict NMs completing a follow-up interview, having a private source of water, was a proxy for CA's socioeconomic status. This may have been because CAs with greater resources may have had more time available to pass information to their NMs, retaining the NM's interest longer. ${ }^{55}$ We also note that less HIV knowledge and living with HIV predicted reduced likelihood of completing a follow-up questionnaire among NMs, which means that those who might have benefitted most from spillover of the intervention were more likely to discontinue their involvement. This does not mean that they did not receive any spillover, only that it was not recorded. Our estimate of the magnitude of the spillover may therefore be biassed towards the null. This is also problematic more generally for interventions of this nature as the very people the intervention aims to benefit may not stay with the programme.

The HIV knowledge gain experienced by the NMs was largely due to the NDE; that is, knowledge spilledover as a result of the CAs participating in NAMWEZA, not because the CAs' HIV knowledge increased, and they passed this new knowledge to their $\mathrm{NM}(\mathrm{s})$. This is most likely because CAs had a high average HIV knowledge score at baseline $(80 \%)$, so there was less room for improvements in knowledge. Following the intervention, CAs' likelihood of complete HIV knowledge did not significantly increase. Therefore, what prompted the increased knowledge of the NMs was the CA becoming empowered through the intervention to pass on their existing knowledge to their NM. ${ }^{56}$

This finding is important for future interventions: spillover effects of this intervention will likely carry over only to those directly-connected to the CAs (opposed to spreading indefinitely in a snowball effect), as the most important thing in transmitting HIV knowledge is receiving the intervention, which only CAs receive. Because NMs do not actually receive the intervention, it is unlikely that they would feel empowered to become CAs themselves (particularly because they will not receive the NAMWEZA sessions), thus limiting the spread of the intervention. For CAs to increase the HIV knowledge of their NMs, they would only need to become empowered to share their information. However, for the NMs to increase the HIV knowledge of their own NMs, they would need to both increase their HIV knowledge, and become empowered to share it. This is less likely to happen than just becoming empowered, and so it is unlikely, though not impossible, that this effect would continue to spread in the population. This may give insight into how to design interventions in the future; if one wants to maximise the number of people who benefit, choosing CAs who form many bridging ties in the community would maximise the potential number of links by which spillover can occur. ${ }^{57}$ Alternatively, interventions can be designed to be selfpropagating; if CAs are empowered to deliver the intervention to others, changing their own NMs into future CAs, those new NMs-turned-CAs could then deliver the intervention to a second set of NMs, again empowering them to become CAs.

Our study is not without limitations. First, there was considerable loss to follow-up of the NMs. Although this actually informed our analysis of the correlates of loss to follow-up, it meant that our analysis of spillover effects may be biassed. Even though the exposure was randomised, the loss to follow-up can result in selection bias if the NMs who left the study were systematically different from those who remained. As we show, the NMs who dropped out were those who would have benefited the most from the intervention because they were more vulnerable and at higher risk than those who did not drop out. Because they are likely to have greater benefits from any spillover effects, we would expect this non-random loss to follow-up to result in an underestimate of the impact of the intervention in the network members. Adjusting for censoring weights may ameliorate this issue. ${ }^{58}$ Second, our use of HRs has important limitations: they are subject to selection bias, are sensitive to study period, and only provide one estimate during the study. ${ }^{43}$ Any of these limitations could affect this analysis, hence our use of logistic regression as a primary analysis. However, they remain useful as a sensitivity analysis. Third, our data did not perfectly fit the requirements of the causal mediation analysis: they were not entirely independent, since multiple NM-CA dyads shared a CA. However, when we randomly removed dyads until there were no repeated CAs, the results were qualitatively very similar, indicating that lack of independence did not unduly affect our results. Fourth, although we were able to tease apart the direct and indirect effects, we are unable to determine the mechanism of the natural direct effect; the data do not allow us to specify whether NMs increased knowledge through speaking to their knowledgeable CA, through researching HIV on their own, or some other mechanism. Future work will have to be done to examine these different pathways.

\section{CONCLUSIONS}

These results have implications for the potential scale-up of the NAMWEZA intervention, as well as future studies and interventions that focus on behavioural interventions in social networks. First, our findings of minimal similarity between CAs and their NMs indicate that this recruitment method allows us to enrol participants from portions of 
the population that are not represented by the CAs alone. Coupling this with a deeper understanding of the mental heuristics CAs used to select NMs (eg, did CAs mentally search their close or peripheral network for those at-risk of HIV), may lead to different strategies for recruitment and retention, leading to stronger effects of behavioural interventions. The mediation analysis presents a compelling picture of how best to ensure the benefits of interventions reach as many people beyond the study participants as possible. Participation of CAs in the intervention resulted in positive effects on their immediate network members' HIV knowledge regardless of how the CAs responded to the intervention. While improvement in HIV knowledge may not necessarily translate to increased safe sex behaviour, it can be seen as a rate-limiting step towards reducing HIV transmission since adequate knowledge is usually necessary for reducing risk behaviour. Future work should examine the exact mechanisms of spillover discussed here, as that is an important clarification that could benefit future studies. Specifically, a similar setup to the study here combined with semi-structured interviews with CAs and NMs about their interactions with one another would help elucidate exactly how NMs increased their HIV knowledge (this population would likely need to comprise CAs who have disclosed their HIV status in order to prevent accidental disclosure). Future work should also examine whether increases in HIV knowledge translate to changes in behaviours which may increase one's risk of contracting HIV, particularly in subSaharan Africa. The results presented herein may inform approaches for increasing participation and potentially conferring greater benefits related to spillover effects in future HIV behavioural interventions.

\author{
Author affiliations \\ ${ }^{1}$ Center for Health Care Innovation, University of Pennsylvania Perelman School of \\ Medicine, Philadelphia, Pennsylvania, USA \\ ${ }^{2}$ CHERP, Philadelphia VAMC, Philadelphia, Pennsylvania, USA \\ ${ }^{3}$ Global Health and Social Medicine, Harvard Medical School, Boston, \\ Massachusetts, USA \\ ${ }^{4}$ Epidemiology, London School of Hygiene and Tropical Medicine, London, UK \\ ${ }^{5}$ School of Medicine, Muhimbili University of Health and Allied Sciences, Dar es \\ Salaam, Tanzania \\ ${ }^{6}$ University of Texas School of Public Health, Austin, Texas, USA \\ ${ }^{7}$ Population Health, London School of Hygiene and Tropical Medicine, London, UK \\ ${ }^{8}$ Management and Development for Health, Dar es Salaam, Tanzania \\ ${ }^{9}$ Biostatistics, Harvard T.H. Chan School of Public Health, Boston, Massachusetts, \\ USA
}

\section{Twitter Jeffrey Rewley @JeffLienert}

Acknowledgements The authors would like to thank the study participants and staff for their time, hard work and dedication. We would also like to thank Guy Harling for their comments on the manuscript.

Contributors JR, MCSF and JPO designed the analysis plan and drafted the initial paper. JR, MCSF, KM, SK, IAL and JT conducted the underlying randomised trial, and designed it to incorporate social networks data and spillover information; these same authors provided feedback on the proposed analysis plan. JR and YL conducted analyses for the paper. All authors helped revise the drafts of the paper and approved the final manuscript.

Funding The authors received funding from the Centers for Disease Control and Prevention via grant CZ/UG.08.0147, the President's Emergency Plan for AIDS Relief
- Tanzania and the UTHealth Innovation for Cancer Prevention Research Training Program Pre-doctoral Fellowship (Cancer Prevention and Research Institute of Texas grant RP160015).

\section{Competing interests None declared.}

Patient and public involvement Patients and/or the public were not involved in the design, or conduct, or reporting, or dissemination plans of this research.

\section{Patient consent for publication Not required.}

Ethics approval All procedures performed in studies involving human participants were in accordance with the ethical standards of the institutional and/or national research committee and with the 1964 Helsinki declaration and its later amendments or comparable ethical standards. Institutional Review Board approval was obtained from Harvard Medical School, the Muhimbili University of Health and Allied Sciences and the National Institute for Medical Research in Tanzania. Particular attention was paid to eliminate the risk of inadvertent disclosure of change agents HIV status to network members.

Provenance and peer review Not commissioned; externally peer reviewed.

Data availability statement No data are available. There are no additional data available. As the data contain enough information to potentially-uniquely identify specific participants, in the sensitive context of HIV, we have chosen not to make the data available.

Open access This is an open access article distributed in accordance with the Creative Commons Attribution Non Commercial (CC BY-NC 4.0) license, which permits others to distribute, remix, adapt, build upon this work non-commercially, and license their derivative works on different terms, provided the original work is properly cited, appropriate credit is given, any changes made indicated, and the use is non-commercial. See: http://creativecommons.org/licenses/by-nc/4.0/.

\section{ORCID iDs}

Jeffrey Rewley http://orcid.org/0000-0002-8534-8377

Sylvia Kaaya http://orcid.org/0000-0001-6732-3590

\section{REFERENCES}

1 Naghavi M, Abajobir AA, Abbafati C, et al. Global, regional, and national age-sex specific mortality for 264 causes of death, 19802016: a systematic analysis for the global burden of disease study 2016. Lancet 2017;390:1151-210.

2 Crepaz N, Tungol-Ashmon MV, Higa DH, et al. A systematic review of interventions for reducing HIV risk behaviors among people living with HIV in the United States, 1988-2012. AIDS 2014;28:633-56.

3 Arnold EA, Kegeles SM, Pollack LM, et al. A randomized controlled trial to reduce HIV-related risk in African American men who have sex with men and women: the Bruthas project. Prev Sci 2019;20:115-25.

4 García de Olalla P, Knobel H, Carmona A, et al. Impact of adherence and highly active antiretroviral therapy on survival in HIV-infected patients. J Acquir Immune Defic Syndr 2002;30:105-10.

5 Joint United Nations Programme on HIV/AIDS and Joint United Nations Programme on HIV/Aids and others. 90-90-90: an ambitious treatment target to help end the AIDS epidemic. Geneva: UNAIDS, 2014.

6 Fisher JD, Fisher WA. Changing AIDS-risk behavior. Psychol Bull 1992;111:455-74.

7 McNeill C, George N, Glover R. An evaluation of sisters informing healing living empowering: increasing HIV knowledge among African American adolescent females using an evidence-based HIV prevention intervention. J Dr Nurs Pract 2017;10:4-10.

8 Carey MP, Braaten LS, Maisto SA, et al. Using information, motivational enhancement, and skills training to reduce the risk of HIV infection for low-income urban women: a second randomized clinical trial. Health Psychol 2000;19:3-11.

9 Sinclair B, McConnell M, Green DP. Detecting spillover effects: design and analysis of multilevel experiments. Am J Pol Sci 2012;56:1055-69.

10 Benjamin-Chung J, Arnold BF, Berger D, et al. Spillover effects in epidemiology: parameters, study designs and methodological considerations. Int J Epidemiol 2018;47:332-47.

11 Truelove HB, Carrico AR, Weber EU, et al. Positive and negative spillover of pro-environmental behavior: an integrative review and theoretical framework. Global Environmental Change 2014;29:127-38.

12 Ghosh D, Krishnan A, Gibson B, et al. Social network strategies to address HIV prevention and treatment continuum of care among at- 
risk and HIV-infected substance users: a systematic scoping review. AIDS Behav 2017;21:1183-207.

13 Benjamin-Chung J, Abedin J, Berger D, et al. Spillover effects on health outcomes in low- and middle-income countries: a systematic review. Int J Epidemiol 2017;46:1251-76.

14 Self S, Grabowski R. Factors influencing knowledge of HIV/AIDS in Nepal: role of socioeconomic interactions. Journal of Social and Economic Development 2018;20:174-91.

15 Banerjee A, La Ferrara E, Orozco-Olvera VH. The entertaining way to behavioral change: fighting HIV with MTV. The World Bank, 2019.

16 Christakis NA, Fowler $\mathrm{JH}$. The spread of obesity in a large social network over 32 years. N Engl J Med 2007;357:370-9.

17 Perkins JM, Subramanian SV, Christakis NA. Social networks and health: a systematic review of sociocentric network studies in lowand middle-income countries. Soc Sci Med 2015;125:60-78.

18 Coleman J, Katz E, Menzel H. The diffusion of an innovation among physicians. Sociometry 1957;20:253-70.

19 VanderWeele TJ. Inference for influence over multiple degrees of separation on a social network. Stat Med 2013;32:591-6. discussion 597-9.

20 Lienert J, Marcum CS, Finney J, et al. Social influence on 5-year survival in a longitudinal chemotherapy ward co-presence network. Netw Sci 2017:5:308-27.

21 Hughes JJ. Paying injection drug users to educate and recruit their Peers: why participant-driven interventions are an ethical public health model. Qual Manag Health Care 1999;7:4-12.

22 Ssali S, Wagner G, Tumwine C, et al. Hiv clients as agents for prevention: a social network solution. AIDS Res Treat 2012;2012:1-7.

23 Cislaghi B, Denny EK, Cissé M, et al. Changing Social Norms: the Importance of "Organized Diffusion" for Scaling Up Community Health Promotion and Women Empowerment Interventions. Prev Sci 2019;20:936-46.

24 Magnani R, Sabin K, Saidel T, et al. Review of sampling hard-toreach and hidden populations for HIV surveillance. AIDS 2005;19 Suppl 2:S67-72.

25 Smith R, Rossetto K, Peterson BL. A meta-analysis of disclosure of one's HIV-positive status, stigma and social support. AIDS Care 2008;20:1266-75.

26 Watters JK, Biernacki P. Targeted sampling: options for the study of hidden populations. Soc Probl 1989;36:416-30.

27 Neema S, Atuyambe LM, Otolok-Tanga E, et al. Using a clinic based creativity initiative to reduce HIV related stigma at the infectious diseases Institute, Mulago national referral Hospital, Uganda. Afr Health Sci 2012;12:231-9.

28 Smith Fawzi MC, Siril H, Liu Y, et al. Agents of change among people living with HIV and their social networks: stepped-wedge randomised controlled trial of the NAMWEZA intervention in Dar es Salaam, Tanzania. BMJ Glob Health 2019;4:e000946.

29 Kaaya S, Arthur G, Siril H, et al. Leadership of PLWH as agents of change: comparing HIV-related risk behavior of people attending art clinics in Dar ES Salaam with members of their social networks. 20th International AIDS Conference, 2014.

30 Brown CA, Lilford RJ. The stepped wedge trial design: a systematic review. BMC Med Res Methodol 2006;6:54.

31 Zou J, Yamanaka Y, John M, et al. Religion and HIV in Tanzania: influence of religious beliefs on HIV stigma, disclosure, and treatment attitudes. BMC Public Health 2009;9:75.

32 McCarthy B. Appreciative inquiry: an alternative to behaviour management. Dementia 2017;16:249-53.

33 Siril HN, Kaaya SF, Smith Fawzi MK, et al. Clinical outcomes and loss to follow-up among people living with HIV participating in the NAMWEZA intervention in Dar ES Salaam, Tanzania: a prospective cohort study. AIDS Res Ther 2017;14:18.

34 Carey MP, Schroder KEE. Development and psychometric evaluation of the brief HIV knowledge questionnaire. AIDS Educ Prev 2002;14:172-82.

35 Haile BJ, Chambers JW, Garrison JL. Correlates of HIV knowledge and testing: results of a 2003 South African survey. Journal of Black Studies 2007;38:194-208.
36 Aarø LE, Breivik K, Klepp K-I, et al. An HIV/AIDS knowledge scale for adolescents: item response theory analyses based on data from a study in South Africa and Tanzania. Health Educ Res 2011;26:212-24.

37 Peltzer K, Matseke G, Mzolo T, et al. Determinants of knowledge of HIV status in South Africa: results from a population-based HIV survey. BMC Public Health 2009;9:174.

38 Fedorov V, Mannino F, Zhang R. Consequences of dichotomization. Pharm Stat 2009;8:50-61.

39 Easterly W. How the millennium development goals are unfair to Africa. World Dev 2009;37:26-35.

40 Barbarin OA, Khomo N. Indicators of economic status and social capital in South African townships: what do they reveal about the material and social conditions in families of poor children? Childhood 1997;4:193-222.

41 Smith JA, McPherson M, Smith-Lovin L. Social distance in the United States: sex, race, religion, age, and education homophily among confidants, 1985 to 2004. American Sociological Review 2014;79:432-56

42 Lesko CR, Edwards JK, Cole SR, et al. When to Censor? Am J Epidemiol 2018;187:623-32.

43 Hernán MA. The hazards of hazard ratios. Epidemiology 2010;21:13-15

44 VanderWeele T. Explanation in causal inference: methods for mediation and interaction. Oxford University Press, 2015.

45 Hong G. Causality in a social world: moderation, mediation and spillover. John Wiley \& Sons, 2015.

46 Forastiere L, Mealli F, VanderWeele TJ. Identification and estimation of causal mechanisms in clustered Encouragement designs: disentangling bed nets using Bayesian principal stratification. J Am Stat Assoc 2016;111:510-25.

47 Imai K, Jiang Z. Identification and sensitivity analysis of contagion effects in randomized placebo-controlled trials. J R Stat Soc Ser A Stat Soc 2019;11.

48 Shalizi CR, Thomas AC. Homophily and contagion are generically confounded in observational social network studies. SMR 2012;40:211-39.

49 de la Haye K, Robins G, Mohr P, et al. Homophily and contagion as explanations for weight similarities among adolescent friends. $J$ Adolesc Health 2011;49:421-7.

50 Vanderweele TJ, Hong G, Jones SM, et al. Mediation and spillover effects in group-randomized trials: a case study of the 4Rs educational intervention. J Am Stat Assoc 2013;108:469-82.

51 Valeri L, Vanderweele TJ. Mediation analysis allowing for exposuremediator interactions and causal interpretation: theoretical assumptions and implementation with SAS and SPSS macros. Psychol Methods 2013;18:137-50.

52 Leung MP. Treatment and spillover effects under network interference. Review of Economics and Statistics 2016:1-42.

53 Latkin CA, Davey-Rothwell MA, Knowlton AR, et al. Social network approaches to recruitment, HIV prevention, medical care, and medication adherence. J Acquir Immune Defic Syndr 2013;63 Suppl 1:S54-8.

54 Galea S, Tracy M. Participation rates in epidemiologic studies. Ann Epidemiol 2007;17:643-53.

55 Berkman LF, support S, networks social. Social support, social networks, social cohesion and health. Soc Work Health Care 2000;31:3-14.

56 Langford CP, Bowsher J, Maloney JP, et al. Social support: a conceptual analysis. J Adv Nurs 1997;25:95-100.

57 Schneider JA, Zhou AN, Laumann EO. A new HIV prevention network approach: sociometric peer change agent selection. Soc Sci Med 2015;125:192-202.

$58 \mathrm{He}$, Eriksson F, Scheike TH, et al. A proportional hazards regression model for the Sub-distribution with covariates adjusted Censoring weight for competing risks data. Scand Stat Theory Appl 2016;43:103-22. 